\title{
Mortality Rate and Causes of Death in Children Aged 1-59 Months in Northeastern Iran During 2011-2017
}

\author{
(1) Mehdi Safarpour¹, (1) Amir Tiyuri2, (1) Parvin Mohamadzade3, (1) Maryam Dadjoo3, \\ (1) Seyed Ahmad Hashemy33, (1) Masume Mohamadzade ${ }^{4}$, (1) Ehsan Shahrestanaki ${ }^{5}$ \\ 1Iran University of Medical Sciences, Faculty of Public Health, Department of Epidemiology, Tehran, Iran \\ 2 Student Research Committee, Iran University of Medical Sciences, Tehran, Iran \\ ${ }^{3}$ Health Deputy, North Khorasan University of Medical Sciences, Bojnurd, Iran \\ ${ }^{4}$ Health Deputy, Babol University of Medical Sciences, Babol, Iran \\ ${ }^{5}$ Tehran University of Medical Sciences, Faculty of Public Health, Department of Epidemiology and Biostatistics, Tehran, Iranw
}

\begin{abstract}
Aim: The mortality rate indicator for children is one of the important indicators of population health and socioeconomic development of countries. In addition, to reduce the mortality in children, it is essential to determine causes of death. This study aims to determine the mortality causes in children aged 1-59 months in Northeastern Iran.

Materials and Methods: This population-based cross-sectional study was conducted based on data from the child mortality surveillance system, from March 2011 to March 2017. The mortality rate was evaluated according to demographic variables (sex, place of residence and mother's education). Causes of death were coded according to the International Classification of Diseases. Chi-square and Mann-Whitney $U$ tests were applied to investigate the relationships.

Results: The mortality rate in children aged 1-59 months was 8.2 per 1,000 live births (CI 95\%: 7.6-8.8) from 2011 to 2017 . Totally $53.2 \%$ of mortalities occurred in boys ( $p=0.07$ ). The frequency of death in the age group of 1-11 months was 1.7 times more than the age group of $12-59$ months. The highest mortality rates occurred in rural areas and among children with illiterate mothers. During the study period, on average, the mortality rate decreased by 0.7 per 1,000 live births every year. The most common causes of death were unintentional accidents ( $33.8 \%$ ) and congenital or chromosomal abnormalities (22.1\%).
\end{abstract}

Conclusion: Improving maternal education is among the key strategies for reducing child mortality. Therefore, it is necessary to raise the level of education and awareness regarding childhood mortality, especially in rural areas.

Keywords: Children, mortality rate, causes of death, maternal education, Iran

\section{Introduction}

The mortality rate for children has received more attention as an indicator of population health and a country's development especially in the last decade (1).
This issue holds great importance which has led to the Millennium Development Goals (MDGs) aim to reduce child mortality with a target of reducing under-five mortality rates by two thirds over the period 1990-2015 (2). The child 
mortality rate in communities has been decreased due to economic and social developments and carrying out effective interventions (3). According to the World Health Organization (WHO), substantial global progress has been made in reducing child deaths since 1990. Worldwide, the number of under-5 deaths declined from 12.5 million in 1990 to 5.6 million in 2016. In other words, 35,000 deaths per day in 1990 compared with 15,000 in 2016. During these years, the under- 5 mortality rate dropped by $56 \%$ worldwide, in other words, 93 deaths per 1,000 live births in 1990 to 41 in 2016 (4). According to the results of the global burden of disease study, lower respiratory infections (20.1\%), diarrheal diseases (17.4\%), malaria (11.8\%), other no communicable diseases (8.1\%), and nutritional deficiencies $(6.7 \%)$, were reported to be the most important causes of death in children aged 1-11 months. Furthermore, malaria $(20.8 \%)$, lower respiratory infections (12.4\%), diarrhea $(11.9 \%)$, chronic non-communicable diseases (9.9\%) and nutritional disorders (7.2\%) were the most prevalent causes of death among children aged 1-4 years old (5). According to the $\mathrm{WHO}$, more than half of these early child deaths are due to conditions that could be prevented or treated with access to simple, affordable interventions. Strengthening health systems to provide such interventions to all children will save many young lives (4). The results of a variety of studies in Iran indicate that the under-five mortality rate has decreased in recent years $(6,7)$. Given that reducing the mortality rate of this age group has been consistent with international goals as a national and academic priority, and due to the lack of data in this regard, this study was conducted to determine the mortality rate and causes of death in children aged 1-59 months in North Khorasan province, northeastern Iran, from March 2011 to March 2017.

\section{Materials and Methods}

In this population-based cross-sectional study, we considered all actual and recorded deaths through the child mortality surveillance system, from March 2011 to March 2017, to investigate the mortality rate and causes of death in children aged 1-59 months in North Khorasan Province, Located in Northeastern Iran, which had a population of 863,092 according to the 2016 national census. This province covers an area of 28,434 square kilometers and contains 8 counties with Bojnurd as its provincial capital. Due to the importance given to the registering of child mortality in the health care system of Iran, all deaths should be reported and reviewed by the child death committee. Therefore, the database of child deaths in the health department is a comprehensive and valid source. Iranian nationality and residence in North Khorasan province were the eligibility criteria, in contrast, inadequate and misleading information were considered as exclusion criteria (however, there were no missed cases). In this study, we performed a secondary analysis of the data collected by the health department of North Khorasan University of Medical Sciences. These data were obtained from the health department de-identified and without the confidential information of individuals. Therefore, informed parental consent was not obtained due to the design of this study.

The data needed for this study including gender, age, place of residence, mother's education and cause of death were extracted from the standard checklist of the Children's Health Department of the Ministry of Health in the health department of North Khorasan University of Medical Sciences. This checklist investigates the history of each child death since the beginning of the first signs of risk to death and includes four sections: an overview of family health status, outpatient medical measures, hospitalized medical measures and a specialized checklist examines the cause of the death of each child aged 1 to 59 months, which is completed by a Pediatrician after reviewing the previous checklists. The process of recording and reporting a child's mortality is as follow: when the death has occurred in the hospital, the main criterion for the leading cause of death in hospitals is under the supervision of the death committee, but when the death is reported by a physician at a health center in cases where death occurred outside the hospital, reports were examined by relevant experts at the health department and causes of death were coded according to the International Classification of Diseases. The mortality rate was estimated in each year as deaths which occurred in the age group of 1-59 months per 1,000 live births. To report the results, the frequency was used to describe qualitative variables and the mean and $95 \% \mathrm{Cl}$ for quantitative variables. Pie charts are used to display the causes of death in each age group (1-11 months and 12-59 months).

\section{Statistically Analysis}

The frequency of mortality is represented in a table according to demographic variables. Chi-square and MannWhitney $U$ tests were applied to examine the relationship between qualitative variables and comparison of median age at death. The statistical significance level was set at less than 0.05. Additionally, the mortality trend was also investigated using linear regression, which was the line gradient considered as the average of the changes in mortality during the period. 
Table I. Distribution of deaths by demographic characteristics

\section{Age groups (months)}

\begin{tabular}{|c|c|c|c|c|}
\hline Variable & $1-11$ & $12-59$ & $1-59$ & $\mathbf{p}$ \\
\hline \multicolumn{4}{|c|}{ Gender n (\%) } & \multirow{3}{*}{0.07} \\
\hline Male & $256(52.0)$ & $158(55.2)$ & $414(53.2)$ & \\
\hline Female & $236(48.0)$ & $128(44.8)$ & $364(46.8)$ & \\
\hline \multicolumn{4}{|c|}{ Place of residence $\mathrm{n}(\%)$} & \multirow{3}{*}{$<0.001$} \\
\hline Urban & $149(30.3)$ & $82(28.7)$ & $231(29.7)$ & \\
\hline Rural & $343(69.7)$ & $204(71.3)$ & $547(70.3)$ & \\
\hline \multicolumn{4}{|c|}{ Mother's education n (\%) } & \multirow{6}{*}{$<0.001$} \\
\hline Illiterate & $159(32.3)$ & $88(30.8)$ & $247(31.7)$ & \\
\hline Elementary & $134(27.2)$ & $97(34.0)$ & $231(29.7)$ & \\
\hline Secondary & $106(21.5)$ & $48(16.7)$ & $154(19.8)$ & \\
\hline High school & $73(14.8)$ & $42(14.6)$ & $115(14.8)$ & \\
\hline Academic & $20(4.2)$ & $11(3.9)$ & $31(4.0)$ & \\
\hline
\end{tabular}

a.

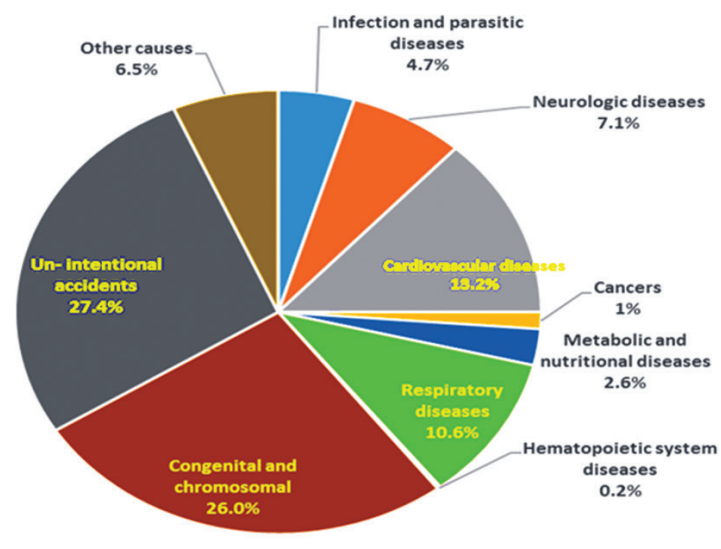

b.

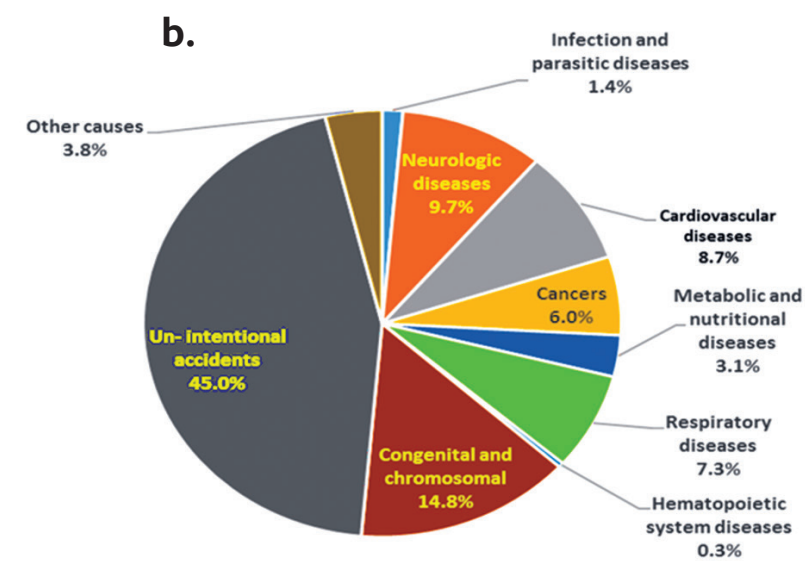

Infection and
parasitic disease

c.

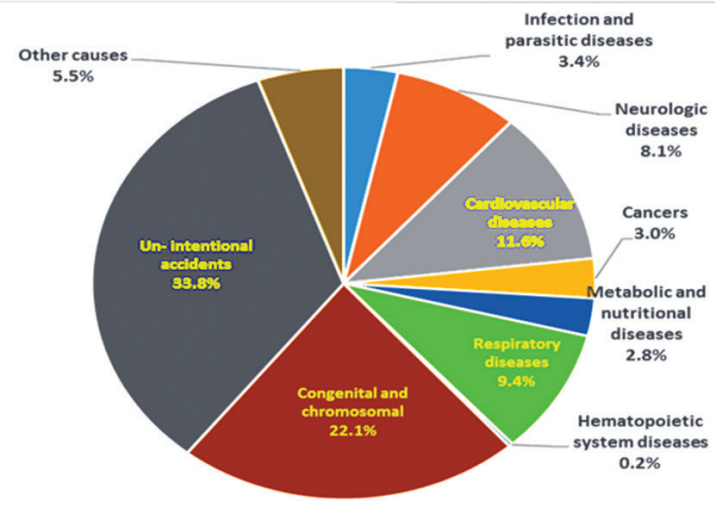

Figure 1. a) Distribution of deaths among children age 1-11 months, by cause. b) Distribution of deaths among children age 12-59 months, by cause. c) Distribution of deaths among children aged 1-59 months, by cause

\section{Results}

According to the table I, 778 deaths were recorded in children aged 1-59 months and that 414 (53.2\%) of them occurred in boys and $364(46.8 \%)$ in girls $(p=0.07)$. Totally, $63.2 \%$ of deaths (492 cases) occurred in the age group of 1-11 months. In other words, the mortality rate in the age 


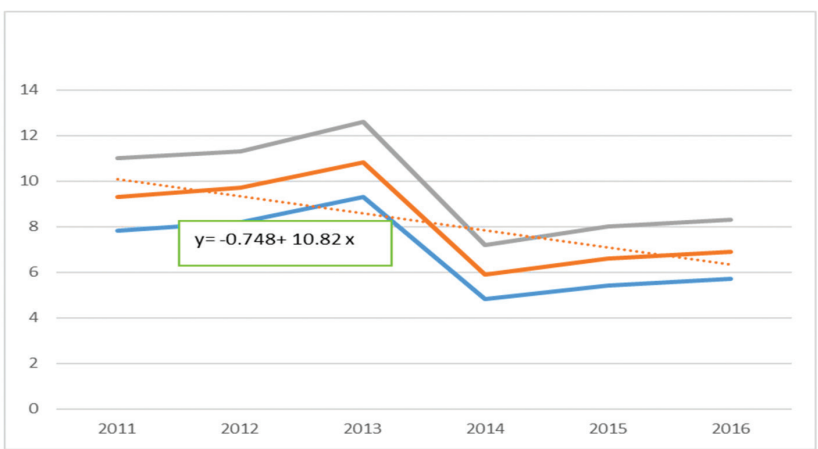

Figure 2. Trend of mortality rate with confidence interval $95 \%$ children 1-59 months in the study period

group of less than one year (1-11 months) was 1.7 times more than the age group of 12-59 months. The median age at death for boys and girls was 7 months (Interquartile: 16.0) and 6 months (Interquartile: 14.3$)$ respectively $(p=0.8)$. The childhood mortality in the rural areas (547 cases) was 2.3 times more than in urban areas $(p<0.001)$. This significant difference was observed in both age groups and death cases in rural areas in the age groups of 1-11 and 12-59 months was 2.3 and 2.4 times more than urban areas respectively. The mortality rate of children with illiterate mothers was higher than children with mothers with elementary and academic education $(p<0.001)$. In addition, $31.7 \%$ of mothers were illiterate and $81.3 \%$ of them lived in rural areas. Regarding the records of 94,501 new birth cases during the study period, totally, the mean annual mortality rate for children was estimated to be 8.2 per 1,000 live births $(95 \% \mathrm{Cl}$ : 7.6-8.8). Figure 1 show the causes of death in age groups. Unintentional accidents, congenital and chromosomal abnormalities, cardiovascular diseases, respiratory diseases, and nervous system diseases are noted as the top five leading causes of death in the age group of 1-11 months. The top five leading causes of death in the age group of 12-59 months were unintentional accidents, congenital and chromosomal abnormalities, nervous system diseases, cardiovascular diseases, and respiratory diseases respectively. Also, in the top ten causes of death, the median of age of death was lower than 12 months except for cancers and hematopoiesis system diseases.

Figure 2 indicates the mortality rate from 2011 to 2017; the highest and lowest mortality rates occurred during 2013 and 2014, with 10.8 and 5.9 deaths per 1,000 live births, respectively. From 2011 to 2017, the mortality rate declined in the age group of 1-59 months, from 9.3 deaths per 1,000 live births in 2011 to 6.9 in 2017. Figure 3 shows that the frequency of deaths for boys was higher than for girls in the study period, except in 2013 and 2016.

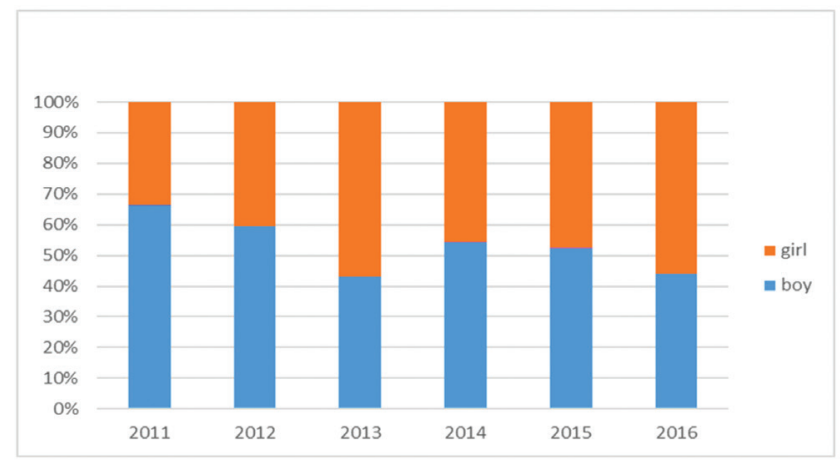

Figure 3. Frequency of deaths among children aged 1-59 months by gender in the study period

\section{Discussion}

The findings of the present study suggests that child mortality in rural area was higher than urban areas. In contrast, according to a study of Izadi et al.(8) in Kermanshah province, child mortality was higher in urban areas. Also, according to the results of the present study, unintentional accidents were the leading cause of death in children aged 1-59 months, whereas Rahbar et al. (7) in their study reported that the most common cause of mortality were the congenital and chromosomal abnormalities in Iran.

With the end of the era of the MDGs, the international community agreed on a new framework, the sustainable development goals (SDGs). The SDGs target for child mortality represents a renewed commitment to the world's children: with an end date of 2030, all countries aim to reduce neonatal mortality to at least as low as 12 deaths per 1,000 live births and under-five mortality to at least as low as 25 deaths per 1,000 live births for preventable mortality (9). In the last few decades, childhood mortality rates have decreased in most countries (10). Despite the significant improvements made in reducing child mortality, child survival remains an urgent concern. In spite of the availability of interventions to preserve life, it is unacceptable that 15,000 children die from preventable and treatable diseases every day. Furthermore, according to the 6th development plan of the Islamic Republic of Iran, by 2021, the aim is to reduce the mortality rate of children under five years to at least as low as 11.3 deaths per 1,000 live births, while this rate was 15.1 according to the latest report of the Ministry of Health in 2017 (11). The results of the present study showed that there was a decline in the trend of mortality in children aged 1-59 months in the North Khorasan province, which is consistent with the results of other studies in other provinces of Iran $(8,12,13)$. Although the results of the study indicated that there is a relative success in achieving the SDGs, unfortunately, the death rate of children aged 1-59 in 
this province is higher than the national average (5.1 deaths per 1,000 live births) and the second lowest rank belongs to this province after Sistan and Baluchestan province (11). Soori et al. (6) compared the rates and causes of under-five mortality in Iran with the Eastern Mediterranean region and the world. Between 1990 and 2012, the under-five mortality rate in Iran dropped from 56 deaths per 1,000 live births to 18 deaths per 1,000 live births, and so ranked 11th among the 21 Eastern Mediterranean countries.

Based on the results, the death rate reached its highest level (10.8 deaths per 1,000 live births) in 2013. Under further scrutiny, it was found that the main contributing cause was due to the increase in central nervous system diseases and opioid poisoning along with traffic accidents compared to the previous year. In contrast, this rate unexpectedly dropped by $46 \%$ in the next year ( 5.8 deaths per 1,000 live births). This decline perhaps was due to low reporting or a lack of sufficient sensitivity of the health system in recording deaths due to shifting and relocation of experts on children's programs at health centers and health department. As well as this, the reduction may be actual and attributable to the effect of the rapid interventions by the health system such as increasing awareness, improvements in the care system and so on, which should be specifically investigated in other studies.

According to the study of Frey and Field (14), improvements in socioeconomics, education, housing, nutrition, health care, and sanitation have contributed to reducing childhood mortality. According to the results, a major part of the deaths occurred in rural areas, which is consistent with the results of previous studies $(7,15)$. Koffi et al. (16) in their study entitled the social determinants of mortality among children aged 1-59 months reported that three quarters of deaths occurred in rural areas. Based on another study in Iran (17), the mortality for children in rural areas was higher than in urban areas. Unequal income distribution and facilities between the rural and urban areas which may affect mortality in children through a number of factors including low maternal education, malnutrition in both the mother and child, a lack of proper access to health services and facilities especially during pregnancy and the low quality of medical services particularly infant medical care can account for the difference between these two areas (18). In addition, the results of the present study showed that the educational level of mothers in rural areas was lower than in urban areas. Evidence suggests that girls with minimal primary education when they reach adulthood, compared to illiterate girls, are likely to better manage their desired family size based on their capacity and there is a strong probability that they provide better care for their children (19). The distribution of mother's educational levels among deceased children in the present study showed that more than three quarters of deaths were found in children of mothers who were illiterate mothers or only had elementary education, which is consistent with the results of other studies $(7,15,17)$. It has been proven by several studies that maternal education plays an important role in increasing health awareness, adopting appropriate fertility behaviors, the better use of health services related to pregnancy, child nutrition, and health and medical care $(20,21)$. Similar to the results of other studies, the frequency of deaths in boys was higher than in girls in our study $(8,15-$ 17). According to some previous studies, gender differences in child mortality are due to complex interactions of biological, genetic, social, and environmental factors $(22,23)$. However, the causes of gender differences in childhood mortality can be explained by two biological and environmental mechanisms. According to the biological perspective, higher rates of mortality in boys can be attributed to their poor biological structure (22) that was confirmed by a report from the United Nations (23) on the existence of some biological factors favorable to female survival compared to males. Girls are less vulnerable to perinatal conditions, such as intrauterine injuries, intrauterine hypoxia, choking, prematurity, Respiratory Distress syndrome, intestinal and lower respiratory tract infections compared to boys. In the environmental factors approach, there is the emphasis on modifiable external factors that affect under-five morbidity and mortality, including mother and infant feeding, gender preference, infant care, breastfeeding, environmental health threats at home and socioeconomic levels (24). In our study, five factors including unintentional accidents, congenital and chromosomal anomalies, cardiovascular diseases, respiratory diseases, and neurological diseases have been noted as the leading causes of death in children aged 1 to 59 months. In addition, more than two-thirds of the deaths were also attributable to the three leading causes of death, and that the contribution of unintentional accidents was 50 percent. Unintentional accidents and congenital and chromosomal anomalies were the main causes of death in both age groups (7). However, according to the 2017 report from the United Nation Inter-agency Group for Child Mortality Estimation, the leading causes of death in this age group were malaria, diarrhea, injury, pneumonia and others (9). In other studies in Iran, similar to the results of the present study, congenital and chromosomal anomalies and unintentional accidents were reported as the leading causes of death in this age group. The low 
mortality rate due to infectious diseases in comparison with some studies reflects the relative success of children's infectious disease prevention programs. Furthermore, since non-communicable diseases became the leading cause of death in children aged 1 to 59 months, it seems that a variety of interventions and strategies are needed to reduce the mortality rate in these children.

\section{Study Limitations}

Despite being population-based and having full coverage of the deaths as the strengths of the present study, our study has several limitations worth noting: the inability to access other variables that affect child death, such as birth weight, birth order, maternal age, pregnancy status, pre and postnatal care and environmental factors including the family's socioeconomic status. Other limitations of this study were due to its retrospective design and the use of secondary data that can affect the data quality as incorrect records may cause misclassification or the low quality of recorded data may lead to classification bias. A further limitation and a potential source of bias in our study was the change in the population of urban and rural areas due to changes in country divisions that made it difficult to calculate the death rate. In addition, the shifting and relocation of experts on children's programs at health centers and health departments is one of the sources of bias that may have affected the quality of data.

\section{Conclusion}

Considering the causes of death among children is very important for the development of health plans, the assessment of ongoing programs and monitoring and prioritizing interventions. Improving maternal education is among the key strategies for reducing childhood mortality. Therefore, it is necessary to raise the level of education and awareness regarding childhood mortality, especially in rural areas.

\section{Acknowledgments}

Words of thanks are offered to the health deputy of North Khorasan University of Medical Sciences for their assistance in this article.

\section{Ethics}

Ethics Committee Approval: In this study, we performed a secondary analysis of the data collected by the health department of North Khorasan University of Medical Sciences. These data were obtained from the health department de-identified and without the confidential information of individuals.
Informed Consent: Informed parental consent was not obtained due to the design of this study.

Peer-review: Externally peer-reviewed

\section{Authorship Contributions}

Concept: M.S., A.T., M.D., E.S., Design: M.S., A.T., P.M., M.M., Data Collection or Processing: P.M., M.D., S.A.H., M.M., Analysis or Interpretation: A.T., E.S., Literature Search: M.S., S.A.H., M.M., Writing: M.S., P.M., M.D.

Conflict of Interest: No conflict of interest was declared by the authors.

Financial Disclosure: The authors declared that this study received no financial support.

\section{References}

1. Murray C), Laakso T, Shibuya K, Hill K, Lopez AD. Can we achieve Millennium Development Goal 4? New analysis of country trends and forecasts of under-5 mortality to 2015. Lancet 2007;370:1040-54.

2. WHO. WHO | Newborn death and illness: World Health Organization; 2011 [Available from: http://www.who.int/ pmnch/media/press_materials/fs/fs_newborndealth_illness/ en/.

3. UNICEF. Levels and Trends in Child Mortality, 1990-2012. Report 2013. 2013.

4. WHO. Children: reducing mortality: $\mathrm{WHO} ; 2018$ [updated October 2017. Available from: http://www.who.int/ mediacentre/factsheets/fs178/en/.

5. Lozano R, Naghavi M, Foreman K, et al. Global and regional mortality from 235 causes of death for 20 age groups in 1990 and 2010: a systematic analysis for the Global Burden of Disease Study 2010. Lancet 2012;380:2095-128.

6. Soori H, Rafiei E, Entezami N, Hasani J, Hossaini SM. A comparison study on rate and causes of under 5 years old deaths in Iran, eastern Mediterranean region and the world. I Safety Promot Injury Prev 2016;4:1-8.

7. Rahbar M, Ahmadi M, Lornejad H, Habibelahi A, Sanaei-Shoar T, Mesdeaghinia A. Mortality causes in children 1-59 Months in Iran. Iran J Public Health 2013;42(Supple1):93.

8. Izadi N, Shetabi H, Bakhtiari S, Janat AM, Parabi M, Ahmadi K. The Rate and Causes of Infant Mortaliry in the Hospitals of Kermanshah Province During 2011-2014. J Rafsanjan Univ Med Sci 2016;15:129-38.

9. UNICEF, WHO, World Bank. Levels and trends in child mortality 2017 [cited 2018. Available from: http://www.who.int/ maternal_child_adolescent/en/-2013/en/pdf.

10. Rajaratnam JK, Marcus JR, Flaxman AD, et al. Neonatal, postneonatal, childhood, and under-5 mortality for 187 countries, 1970-2010: a systematic analysis of progress towards Millennium Development Coal 4. Lancet 2010;375:1988-2008.

11. Health Department-Population and Family Health Office. Report of the national system of death surveillance of children 1-59 months. Tehran: Ministry of Health of the Islamic Republic of Iran; 2017. 
12. Ahmadi A, Javadi A. Trends and Determinants of Infant Mortality Rate in Fars Province during 2001-2011. J Rafsanjan Univ Med Sci 2015; 14:37-46.

13. Sharifian R, Ghanbari Jahromi M, Khammarnia M, Shokrpour $\mathrm{N}$, Kasani A. The Comparison of Maternal and Child Health Indicators before and after the Family Physician Program in Shiraz, from 2001 to 2012. I Health Sci Surveillance Sys January 2017;5:15-21.

14. Frey RS, Field C. The determinants of infant mortality in the less developed countries: a cross-national test of five theories. Soc Indicat Res 2000;52:215-34.

15. Hossain MM, Mani KK, Islam MR. Prevalence and determinants of the gender differentials risk factors of child deaths in Bangladesh: evidence from the Bangladesh demographic and health survey, 2011. PLoS Neglected Trop Dis 2015;9:e0003616.

16. Koffi AK, Kalter HD, Loveth EN, Quinley J, Monehin J, Black RE. Beyond causes of death: The social determinants of mortality among children aged 1-59 months in Nigeria from 2009 to 2013. PLoS One 2017;12:e0177025.

17. Motlagh ME, Kelishadi R, Barakati SH, Lornejad HR, Poursafa P. Distribution of mortality among 1-59 month-old children across Iranian provinces in 2009: the national mortality surveillance system. Arch Iran Med 2013;16:29.
18. Yi B, Wu L, Liu H, Fang W, Hu Y, Wang Y. Rural-urban differences of neonatal mortality in a poorly developed province of China. BMC Publ Health 2011;11:477.

19. Veneman AM. Education is key to reducing child mortality: the link between maternal health and education. UN Chronicle 2007;44:58-9.

20. Raghupathy S. Education and the use of maternal health care in Thailand. Soc Sci Med 1996;43:459-71.

21. Kravdal $\varnothing$. Child mortality in India: the community-level effect of education. Popul Stud 2004;58:177-92.

22. Pongou R. Why is infant mortality higher in boys than in girls? A new hypothesis based on preconception environment and evidence from a large sample of twins. Demography 2013;50:421-44

23. United Nations. Sex Differentials in Childhood Mortality New York: Department of Economic and Social Affairs Population Division; 2011 [cited 2018. Available from: http:// www.un.org/esa/population/publications/SexDifChildMort/ SexDifferentialsChildhoodMortality.pdf.

24. Rosenstock S, Katz J, Mullany LC, et al. Sex differences in neonatal mortality in Sarlahi, Nepal: the role of biology and environment. J Epidemiol Community Health 2013;67:986-91. 